Discussion Paper No. 02-14

Die Ökologische Steuerreform in Deutschland

- ein umweltpolitisches Feigenblatt

Christoph Böhringer und Robert Schwager

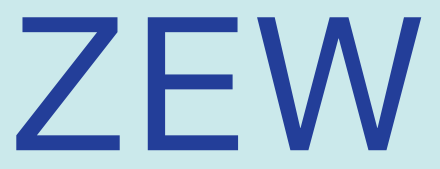

Zentrum für Europäische Wirtschaftsforschung GmbH

Centre for European

Economic Research 
Discussion Paper No. 02-14

\title{
Die Ökologische Steuerreform in Deutschland \\ - ein umweltpolitisches Feigenblatt
}

\author{
Christoph Böhringer und Robert Schwager
}

Download this ZEW Discussion Paper from our ftp server:

ftp://ftp.zew.de/pub/zew-docs/dp/dp0214.pdf

Die Discussion Papers dienen einer möglichst schnellen Verbreitung von neueren Forschungsarbeiten des ZEW. Die Beiträge liegen in alleiniger Verantwortung der Autoren und stellen nicht notwendigerweise die Meinung des ZEW dar.

Discussion Papers are intended to make results of ZEW research promptly available to other economists in order to encourage discussion and suggestions for revisions. The authors are solely responsible for the contents which do not necessarily represent the opinion of the ZEW. 


\section{Das Wichtigste in Kürze}

Die Ökologische Steuerreform wurde expressis verbis mit der Zielsetzung verabschiedet, einen ökologischen Umbau der Industriegesellschaft einzuleiten und den Arbeitsmarkt zu entlasten. Obwohl das Beschäftigungsziel in Zeiten hoher Arbeitslosigkeit Priorität genießt, ist seine Betonung im Zusammenhang mit der Ökologischen Steuerreform kontraproduktiv. Ökologisch sinnvolle Maßnahmen könnten gesellschaftspolitisch auf Ablehnung stoßen, weil die erhofften Arbeitsmarkteffekte ausbleiben. Da eine substantielle zweite Dividende unwahrscheinlich ist, kämen Umweltsteuern aus dieser Perspektive unter Rechtfertigungsdruck.

Gerade weil die Aussichten auf positive Beschäftigungswirkungen gering sind, ist das ökologische Ziel für die Begründung von Umweltsteuern ausschlaggebend. Umweltsteuern sollen die Umweltqualität erhöhen und müssen dementsprechend konzipiert werden. Eine einheitliche Besteuerung gewährleistet, daß alle Verursacher von Umweltverschmutzung mit den damit verbundenen Kosten konfrontiert werden. Dann wird Umweltschutz dort geleistet, wo er am wenigsten kostet. Aus theoretischer Sicht können Abweichungen von dieser einfachen Regel vorteilhaft sein, wenn globale Umweltexternalitäten im nationalen Alleingang gemindert werden sollen oder wenn Verzerrungen durch andere Steuern bestehen. Für die Praxis sind die aus der Theorie ableitbaren Empfehlungen zur Steuerdifferenzierung jedoch kaum relevant.

Die gegenwärtige Ausgestaltung der Ökosteuer weicht vom Prinzip der einheitlichen Besteuerung erheblich ab. Über weitreichende Ausnahmen wird die ökologische Lenkungswirkung stark abgeschwächt. Umweltschutz wird damit teurer, als er aus gesamtwirtschaftlicher Sicht sein müßte.

Die Verwässerung der Ökologischen Steuerreform ist politisch auf die geringe Zahlungsbereitschaft der Wähler für Umweltschutz und die starke sektorale Konzentration von Anpassungskosten einheitlicher Umweltsteuern zurückzuführen. Ausnahmeregelungen für energieintensive Branchen haben die politischen und ökonomischen Kosten der Ökologischen Steuerreform verringert. Damit wurde aber auch das Umweltziel faktisch aufgegeben. 


\title{
Die Ökologische Steuerreform in Deutschland - ein umweltpolitisches Feigenblatt
}

\author{
Christoph Böhringer und Robert Schwager \\ Zentrum für Europäische Wirtschaftsforschung \\ Postfach 1034 43, 68034 Mannheim \\ boehringer@zew.de, schwager@zew.de
}

\begin{abstract}
We assess the design of the recent environmental tax reform in Germany. Based on fundamental welfare economics we argue that extensive tax reductions and rebates in favour of energy-intensive industries, as provided by the tax reform, substantially water down its cost-effectiveness. The environmental tax reform essentially boils down to a fiscally motivated fuel tax. From a political economy perspective the exemptions can be explained by voters' low willingness-to-pay for the environment and the sectoral concentration of adjustment costs. With respect to green voters, however, the government does not admit that the environmental objective has effectively been abandoned. We conclude that an honest policy should either give up the environmental tax reform frankly or implement it without exemptions.
\end{abstract}





\section{Einleitung}

Seit 1999 wird im Rahmen der Ökologischen Steuerreform Energie in Deutschland schrittweise verteuert und das zusätzliche Steueraufkommen zur Senkung der Rentenversicherungsbeiträge verwendet. Die fünfte und vorläufig letzte Stufe tritt zum 1.1.2003 in Kraft. Mit der Ökologischen Steuerreform soll zum einen die Umweltqualität verbessert und zum anderen die Abgabenlast auf den Faktor Arbeit gesenkt werden. Neben einer ökologischen Lenkung von Wirtschaftsaktivitäten geht es also auch darum, Arbeitsplätze zu schaffen. Die Fortführung der Ökologischen Steuerreform ist allerdings heftig umstritten, weil Uneinigkeit über die angemessene Ausgestaltung und die zu erwartenden Wirkungen besteht.

Vor diesem Hintergrund unterziehen wir die konkrete Umsetzung der Ökologischen Steuerreform in Deutschland einer kritischen Würdigung. Unser Fazit fällt vernichtend aus: Wegen weitreichender Ausnahmeregelungen gerade für energieintensive Branchen ist die Ökologische Steuerreform zu einem umweltpolitischen Feigenblatt verkümmert. Das Umweltziel wurde politökonomischem Opportunismus geopfert. Im wesentlich reduziert sich die Ökologische Steuerreform auf eine fiskalisch motivierte Erhöhung der Benzinsteuer. Auch Beschäftigungsgewinne sind kaum zu erwarten. Dennoch wird die in Deutschland umgesetzte Ökologische Steuerreform als umwelt- und arbeitsmarktpolitischer Erfolg verkauft.

Aus politökonomischer Sicht erklärt die sektorale Verteilung der Anpassungslasten einer konsequenten Umweltpolitik, warum die Ökologische Steuerreform durch Sonder- und Kompensationsregelungen ausgehöhlt wurde. Einheitliche Umweltsteuern würden umweltintensive Sektoren besonders hart treffen. Zudem sind gerade diese Branchen politisch gut organisiert. Die politökonomisch motivierte Vorzugsbehandlung der energieintensiven Branchen verhindert jedoch den Strukturwandel, der notwendigerweise mit einer effizienten Umweltpolitik verbunden ist.

Um unser Urteil zu begründen, entwickeln wir zunächst aus umweltökonomischer und finanzwissenschaftlicher Sicht Kriterien für die effiziente Ausgestaltung einer Ökologischen Steuerreform. An diesen Kriterien wird die in Deutschland gewählte Umsetzung gemessen. Die Diskrepanz zwischen wohlfahrtsökonomischer Theorie und politischer Realität wird dann 
politökonomisch erklärt. Abschliessend fordern wir, die Ökologische Steuerreform entweder offen aufzugeben oder sie konsequent umzusetzen.

\section{Umweltökonomische und finanzwissenschaftliche Kritieren}

\subsection{Die Hypothese der doppelten Dividende}

Die ökologischen Effekte und Arbeitsmarktwirkungen einer Ökologischen Steuerreform werden seit längerem unter dem Schlagwort der doppelten Dividende thematisiert (Goulder 1995). Eine aufkommensneutrale Umschichtung der Steuern und Abgaben vom Faktor Arbeit auf den Faktor Umwelt soll nicht nur Umweltschäden reduzieren, sondern gleichzeitig auch Wirtschaftsleistung und Beschäftigung beleben. Während die erste Dividende von Umweltsteuern - erhöhte Umweltqualität - unumstritten ist, werden die Aussichten auf eine zweite Dividende - Verringerung der Arbeitslosigkeit - eher skeptisch beurteilt. Für sich genommen hemmen zusätzliche Umweltsteuern die wirtschaftliche Aktivität. $\mathrm{Ob}$ der damit einher gehende Beschäftigungsverlust durch die Senkung der Lohnnebenkosten wettgemacht oder sogar überkompensiert werden kann, wird von zahlreichen theoretischen und empirischen Studien in Zweifel gezogen. Kaum jemand erwartet, daß eine Ökologische Steuerreform den Arbeitsmarkt wesentlich entlasten kann (Wissenschaftlicher Beirat beim Bundesministerium der Finanzen 1997).

Umweltsteuern sind also kein arbeitsmarktpolitisches Instrument, sondern müssen durch ökologische Überlegungen begründet werden. Negative Arbeitsmarkteffekte sind andererseits kein Grund, auf Ökosteuern zu verzichten, es sei denn, das Beschäftigungsziel hat einen höheren Stellenwert als das Umweltziel. Diese wissenschaftliche Einschätzung steht im Gegensatz zur öffentlichen Darstellung der Ökologischen Steuerreform durch manche ihrer Protagonisten. Letztere vermitteln fast den Eindruck, als ob Umweltsteuern vorrangig dem Arbeitsmarkt und gar nicht so sehr der Umwelt dienen sollen.

\subsection{Verursachungsgerechte Umweltsteuern}

In einem marktwirtschaftlichen System signalisiert die Umweltsteuer die Knappheit des Gutes Umwelt, das wegen fehlender Eigentumsrechte keinen Preis hat. Damit können die gesamtwirtschaftlichen Kosten schädlicher Umweltnutzungen internalisiert werden. Der Steuersatz muß dazu in Höhe des Grenzschadens der Umweltverschmutzung gewählt 
werden. Wie bei marktfähigen Gütern sollte auch hier für alle Nutzer der gleiche „Preis“, d.h. der gleiche Steuersatz gelten. So hängt beispielsweise der Schaden, der durch eine Tonne Schwefeldioxid verursacht wird, nicht davon ab, wer sie emittiert hat.

Einheitliche Steuern sind nicht notwendigerweise optimal, wenn das Umweltziel - wie im Fall des Klimaschutzes - global ist, aber im Rahmen eines nationalen Alleingangs erreicht werden soll. In der globalen Bilanz müssen den heimischen Emissionssenkungen unter Umständen erhöhte Emissionen im Ausland gegenübergestellt werden. Dieses Phänomen wird als leakage bezeichnet (Oliveira-Martins et al. 1992). Aus Sicht einer offenen Volkswirtschaft gibt es dafür im wesentlichen drei Gründe. Erstens können Importe umweltintensiver Güter die heimische Produktion verdrängen. Zweitens kann es $\mathrm{zu}$ Standortverlagerungen umweltintensiver Industrien ins Ausland kommen. Drittens kann der Nachfragerückgang in emissionsmindernden Ländern $\mathrm{zu}$ fallenden Weltenergiepreisen führen, sodaß die Energienachfrage in Ländern ohne Klimapolitik steigt. Um diese kontraproduktiven Rückwirkungen abzuschwächen, kann es sinnvoll sein, umwelt- und exportintensive Industrien weniger stark zu besteuern. Allerdings verlieren diese Argumente an Bedeutung, wenn sich auch andere wichtige Handelspartner zu ähnlichen nationalen Umweltmaßnahmen verpflichtet haben. Zudem sind regionale Unterschiede in der Umweltregulierung für die meisten umweltintensiven Industrien im Vergleich $\mathrm{zu}$ Unternehmenssteuern oder Arbeitsmarktbedingungen nur ein untergeordneter Standortfaktor. Darüber hinaus ist zu bedenken, daß die Berücksichtigung von leakage in der theoretischen Betrachtung fein differenzierte Steuersätze verlangt, die sich am Emissionsgehalt von Importen und Exporten orientieren (Hoel 1996). Die hierfür erforderlichen Informationen sind in der Realität kaum verfügbar. Schließlich zeigen empirische Untersuchungen, daß leakage-Effekte nur von untergeordneter quantitativer Bedeutung sind (IPCC 2001, S.543). Insgesamt ist also auch bei einer globalen Externalität eine einheitliche Besteuerung vorzuziehen.

Grundsätzlich stellt sich bei einer globalen Externalität die Frage, ob sich ein umweltpolitischer Alleingang eines Landes überhaupt auszahlt. So hat die Emissionsminderung eines einzelnen Landes nur einen geringfügigen Einfluss auf die Stabilisierung des Weltklimas. Nationale Emissionsminderungen verursachen aber spürbare Kosten in den jeweiligen Ländern. Auf Grund nichtkooperativen Verhaltens wird kein bzw. zu wenig Klimaschutz betrieben (Barrett 1994). Es ist aber fraglich, ob diese orthodoxe Sichtweise rationalen Verhaltens die realen Entscheidungsprozesse richtig abbildet. 
Ergebnisse der experimentellen Wirtschaftsforschung deuten darauf hin, daß Fairnesspräferenzen und die Erwartung reziproken Verhaltens das Trittbrettfahrerproblem abschwächen können (Bolton und Ockenfels 2000). Zwar ist eine gewisse Vorsicht geboten, was die Übertragbarkeit der Ergebnisse aus Laborexperimenten auf das Verhalten von Ländern im internationalen Umweltschutz betrifft. Auch die nationalen Verhandlungsführer müssen aber die Präferenzen ihrer Wähler berücksichtigen, welche sich in den experimentellen Ergebnissen niederschlagen. Aus Sicht eines Landes kann es also durchaus sinnvoll sein, eine Vorreiterrolle im internationalen Umweltschutz zu übernehmen (Lange und Vogt 2002).

\subsection{Wechselwirkungen mit anderen Steuern}

Indem Umweltsteuern Marktversagen in Form von externen Effekten korrigieren, führen sie zu einem aus gesamtwirtschaftlicher Sicht besseren Einsatz der Ressourcen. Sieht man jedoch von Externalitäten ab, führen steuerinduzierte Änderungen der Wirtschaftsaktivitäten dazu, daß Ressourcen suboptimal eingesetzt werden, weil sich die Wirtschaftssubjekte nicht mehr an den tatsächlichen Knappheitsrelationen orientieren. Der durch die Steuer verursachte Einkommensverlust übersteigt deshalb das Steueraufkommen. Um diesen Verlust bei gegebenem Finanzbedarf möglichst gering zu halten, sollten die Steuersätze niedrig und damit die Bemessungsgrundlagen breit sein. Außerdem sollten vorrangig solche Aktivitäten besteuert werden, bei denen es kaum Ausweichreaktionen gibt. Der von Steuern ausgelöste Einkommensverlust ist nämlich umso größer, je stärker die induzierten Verhaltensänderungen sind. Wenn Steuern ausschließlich dem Fiskalzweck dienen, sind Umweltsteuern also weniger geeignet als Einkommensteuern oder einheitliche Verbrauchsteuern und würden in einem optimalen Steuersystem nicht verwendet werden.

Nun entsprechen reale Steuersysteme in vieler Hinsicht nicht diesen Effizienzkritieren. So ist es theoretisch nicht auszuschließen, daß die Erhebung und Rückverteilung zusätzlicher Umweltsteuern dazu beitragen könnten, bestehende Steuerverzerrungen abzumildern. Beispielsweise würde eine spezifische $\mathrm{CO}_{2}$-Steuer für Kohle den falschen Produktionsanreizen, die von der offenbar unangreifbaren Kohlesubventionierung in Deutschland ausgehen, entgegenwirken. Selbst eine Differenzierung der Umweltsteuern zwischen verschiedenen Sektoren wäre dann vermutlich vorteilhaft. Angesichts der Vielfalt der Einzelregelungen in real existierenden Steuersystemen kann aber nicht gesagt werden, in welche Richtung die Differenzierung ausfallen sollte. Auch die ausgefeilte Theorie der 
zweitbesten Besteuerung gibt der praktischen Steuerpolitik keine Orientierung. Letztlich können zu jeder noch so speziellen Ausnahmeregelung wirtschafts- und finanzpolitische Restriktionen gefunden werden, die diese Regelung als zweitbest rechtfertigen. Grundsätzlich ist es also aus rein fiskalischer Sicht nicht angebracht, Ineffizienzen des bestehenden Steuersystems über den indirekten Weg zusätzlicher Umweltsteuern abbauen zu wollen. Auch diese Überlegungen zeigen, daß der Einsatz von Umweltsteuern einer umweltpolitischen Rechtfertigung bedarf.

Wenn die ökologische Lenkungswirkung aber das zentrale Ziel von Umweltsteuern ist, sollten die Steuersätze einheitlich gewählt werden. Je anspruchsvoller das Umweltziel ist, um so stärker wiegt das Argument für einheitliche Steuersätze. Wenn eine einheitliche Besteuerung unmöglich ist, dann sollten Vergünstigungen nicht in solchen Bereichen gewährt werden, in denen es günstige Alternativen zu umweltintensiven Verhaltensweisen gibt. Beispielsweise zeichnet sich die energieintensive Industrie in Deutschland durch eine hohe $\mathrm{CO}_{2}$-Intensität und günstige $\mathrm{CO}_{2}$-Vermeidungsoptionen aus. Schon bei kleinen Steuerermäßigungen nehmen die $\mathrm{CO}_{2}$-Emissionen der energieintensiven Industrie wieder erheblich zu. Ein vorgegebenes gesamtwirtschaftliches Emissionsminderungsziel - wie im Rahmen des Kyoto-Protokolls vereinbart - kann nur eingehalten werden, wenn andere Sektoren entsprechend höhere Vermeidungsanstrengungen unternehmen. Dort sind aber die Vermeidungsoptionen kostspieliger, so daß deutlich höhere Steuersätze nötig werden. Die gesamtwirtschaftlichen Kosten des Umweltschutzes steigen. Die Zusatzkosten einer Steuersatzdifferenzierung wären dabei geringer, wenn diese zulasten und nicht zugunsten der energieintensiven Branchen ausfallen würde (Böhringer 2002).

Welche gesamtwirtschaftlichen Folgen mit der Verwirklichung von Umweltschutzzielen verbunden sind, hängt nicht nur von der erhebungsseitigen Ausgestaltung von Umweltsteuern $\mathrm{ab}$, sondern auch von der Verwendung des zusätzlichen Steueraufkommens. Es steht außer Frage, daß die potentiellen Kosten von Umweltschutz sinken, wenn die zusätzlichen Einnahmen im Rahmen einer aufkommensneutralen Reform dazu verwendet werden, um bestehende steuerliche Verzerrungswirkungen abzubauen. Das Steuer- und Transfersystem bietet dafür zahlreiche Ansatzpunkte. In Zeiten hoher Arbeitslosigkeit ist es nachvollziehbar, daß dem Beschäftigungsziel hohe Priorität eingeräumt und vor allem der Faktor Arbeit entlastet wird. Damit wird sichergestellt, daß die negativen Effekte höherer Umweltsteuern auf die Beschäftigung zumindest gemildert werden. Diese Verbindung von Umweltsteuern 
und Arbeitsmarktzielen ist jedoch nicht zwingend. Andere Verwendungen des Aufkommens, etwa zur Förderung von Umweltprojekten oder zur Senkung der Unternehmenssteuerbelastung, können ebenso sinnvoll sein. Es ist letztlich eine Frage der politischen Prioritäten, wofür die durch die Umweltsteuer erhöhten Staatseinnahmen verwendet werden.

\section{Die Ökologische Steuerreform in Deutschland}

Die Bundesregierung hat am 1.4.1999 die Ökologische Steuerreform eingeleitet. Energieverbrauch wurde seitdem schrittweise verteuert. Die zusätzlichen Einnahmen sollten zur paritätischen Senkung der Rentenversicherungsbeiträge verwendet werden. Tabelle 1 weist die Entwicklung der Energiesteuersätze nach dem gegenwärtigen Rechtsstand aus.

Tabelle 1: Energiesteuersätze in Deutschland (Bach et al. 2001)

\begin{tabular}{|c|c|c|c|c|c|c|c|}
\hline \multirow[t]{2}{*}{ Energieträger } & \multirow{2}{*}{$\begin{array}{r}\text { Steuersätze } \\
\text { vor } 1.4 .99 \\
\text { Pf/ME }\end{array}$} & \multicolumn{2}{|c|}{$\begin{array}{l}\text { Energiesteuer je } \\
\text { Mengeneinheit (ME) } \\
\text { jährliche Erhöhung }\end{array}$} & \multicolumn{2}{|c|}{$\begin{array}{c}\text { Kumulierte Erhöhung } \\
1999 \text { bis } 2003\end{array}$} & \multicolumn{2}{|c|}{$\begin{array}{c}\text { Steuerbelastung } \\
\text { insgesamt im Jahr } 2003\end{array}$} \\
\hline & & ab 1.4 .99 & $\begin{array}{l}\text { danach } \\
\text { jährlich }\end{array}$ & $\mathrm{Pf} / \mathrm{ME}$ & $\mathrm{DM} / \mathrm{GJ}$ & $\mathrm{Pf} / \mathrm{ME}$ & $\mathrm{DM} / \mathrm{GJ}$ \\
\hline Kohlen (kg) & - & - & - & - & - & - & - \\
\hline Heizöl schwer ${ }^{1)}(\mathrm{kg})$ & 3,00 & - & $0,50^{2)}$ & 0,50 & 0,12 & 3,50 & 0,85 \\
\hline Heizöl leicht (l) & 8,00 & 4,00 & 0 & 4,00 & 1,12 & 12,00 & 3,37 \\
\hline Erdgas (kWh) & 0,36 & 0,32 & 0 & 0,32 & 0,89 & 0,68 & 1,89 \\
\hline Elektrizität (kWh) & 0,00 & 2,00 & 0,50 & 4,00 & 11,11 & 4,00 & 11,11 \\
\hline Benzin (1) & 98,00 & 6,00 & 6,00 & 30,00 & 9,27 & 128,00 & 39,55 \\
\hline Diesel (l) & 62,00 & 6,00 & 6,00 & 30,00 & 8,38 & 92,00 & 25,71 \\
\hline \multicolumn{8}{|c|}{ 1) Seit 2000 einheitlicher Mineralölsteuersatz für schweres Heizöl. ${ }^{2)}$ Einmalig im Jahr 2000} \\
\hline \multicolumn{8}{|c|}{$\begin{array}{l}\text { Quellen: Gesetz zum Einstieg in die ökologische Steuerreform (Bundesgesetzblatt I, S. 378, 1999), Gesetz zur } \\
\text { Fortführung der ökologischen Steuerreform (Bundesgesetzblatt I, S. 2432, 1999); Berechnungen von } \\
\text { Bach et al. (2001) }\end{array}$} \\
\hline
\end{tabular}

Das Gesetz zur Ökologischen Steuerreform sieht Ausnahmeregelungen der Energiebesteuerung für bestimmte Energieträger und Branchen vor. Beispiele für Sonderregelungen sind:

- Kohle unterliegt nicht der Mineralölsteuer.

- Hocheffiziente Kraft-Wärme-Kopplungsanlagen sind von der Mineralölsteuer ausgenommen. 
- Unternehmen des Produzierenden Gewerbes und der Land- und Forstwirtschaft zahlen für Heizöl, Gas und Strom nur einen ermäßigten Steuersatz in Höhe von 20\% des Regelsatzes. Diese Ermäßigung greift erst oberhalb einer zusätzlichen Steuerbelastung auf Strom- und Heizstoffe von jeweils $511 €$ im Jahr. Bei Strom entspricht dies im Jahr 2002 einer Verbrauchsmenge von 28,6 MWh.

- Unternehmen des Produzierenden Gewerbes erhalten eine Vergütung, sofern ihre Steuer auf Strom und Heizstoffe mehr als $511 €$ pro Kalenderjahr und mehr als das 1,2-fache der Entlastung durch die Senkung der Arbeitgeberanteile zur gesetzlichen Rentenversicherung beträgt. Rückerstattet wird der Differenzbetrag zwischen der gezahlten Energiesteuer und dem 1,2-fachen des Betrags zur Senkung der Arbeitgeberanteile zur Rentenversicherung.

Mißt man die Umsetzung der Ökologischen Steuerreform in Deutschland an den aufgezeigten Kriterien einer effizienten Umweltpolitik, dann fällt ihre Bewertung negativ aus.

Eine offensichtliche Schwäche ist die Wahl der Energie als Steuergegenstand. Nicht Energienutzung an sich ist umweltbelastend, sondern die bei unterschiedlichen Energieumwandlungen freigesetzten spezifischen Schadstoffemissionen. So ist für die Klimaschutzpolitik eine einheitliche Besteuerung des Energiegehalts von Energieträgern gegenüber einer Besteuerung des spezifischen $\mathrm{CO}_{2}$-Gehalts weniger effektiv. Bei einer konsequenten Umsetzung würde der Energieträger Kohle wegen seines hohen Kohlenstoffgehalts sehr stark belastet werden, die Atomenergie dagegen überhaupt nicht. Offensichtlich spielen hier neben dem Klimaschutz auch andere politische Interessen eine Rolle.

Der zentrale Konstruktionsfehler besteht in den weitreichenden Ausnahme- und Kompensationsregelungen. Diese lassen sich anhand eines einfachen Beispiels zur Stromsteuer veranschaulichen. Zur Zeit beträgt der Regelsatz der Stromsteuer 17,90 €/MWh, der ermäßigte Satz 3,60 €/MWh. Verbraucht ein Unternehmen im Jahr 2002 528,6 MWh, müßte es gemäß dem Regelsatz $9461 €(=528,6 \mathrm{MWh} \times 17,90 € / \mathrm{MWh})$ bezahlen. Für die über 28,6 MWh hinausgehende Strommenge kommt jedoch der ermäßigte Satz zur Anwendung. Damit verringert sich die Steuer auf diesen Teil der Strommenge von $8950 €$ auf $1800 €$. Das Unternehmen zahlt also insgesamt nur $2311 €$. Die Lenkungswirkung der Steuer wird durch den niedrigeren Steuersatz erheblich gemindert. Während das Unternehmen unterhalb von 28,6 MWh je reduzierter MWh 17,90€ an Steuern spart, beträgt der Anreiz zur 
Energieeinsparung oberhalb dieses Sockelbetrags nur noch 3,60 €. Dieses Ergebnis wird durch die Kompensationsregelung noch verschärft. Wenn die von diesem Unternehmen geschuldeten Arbeitgeberbeiträge zur Rentenversicherung beispielsweise um $1000 €$ sinken, dann kann sich das Unternehmen die Differenz zwischen der Stromsteuer und dem 1,2-fachen dieser $1000 €$ erstatten lassen. Im konkreten Fall beträgt die Erstattung $1111 €$. Fatal für die ökologische Zielsetzung ist hier, daß eine Energieeinsparung zwar zu einer Senkung der Stromsteuer, aber auch zu einer genau gleich großen Senkung der Erstattung führt. Die Stromsteuer ist für dieses Unternehmen eine Pauschalsteuer, die keinerlei Anreize zu energiesparendem Verhalten setzt.

Der effektive Tarif der Stromsteuer wird in Abbildung 1 illustriert, wo die Steuerzahlung nach Abzug der Erstattung in Abhängigkeit vom Stromverbrauch dargestellt ist.

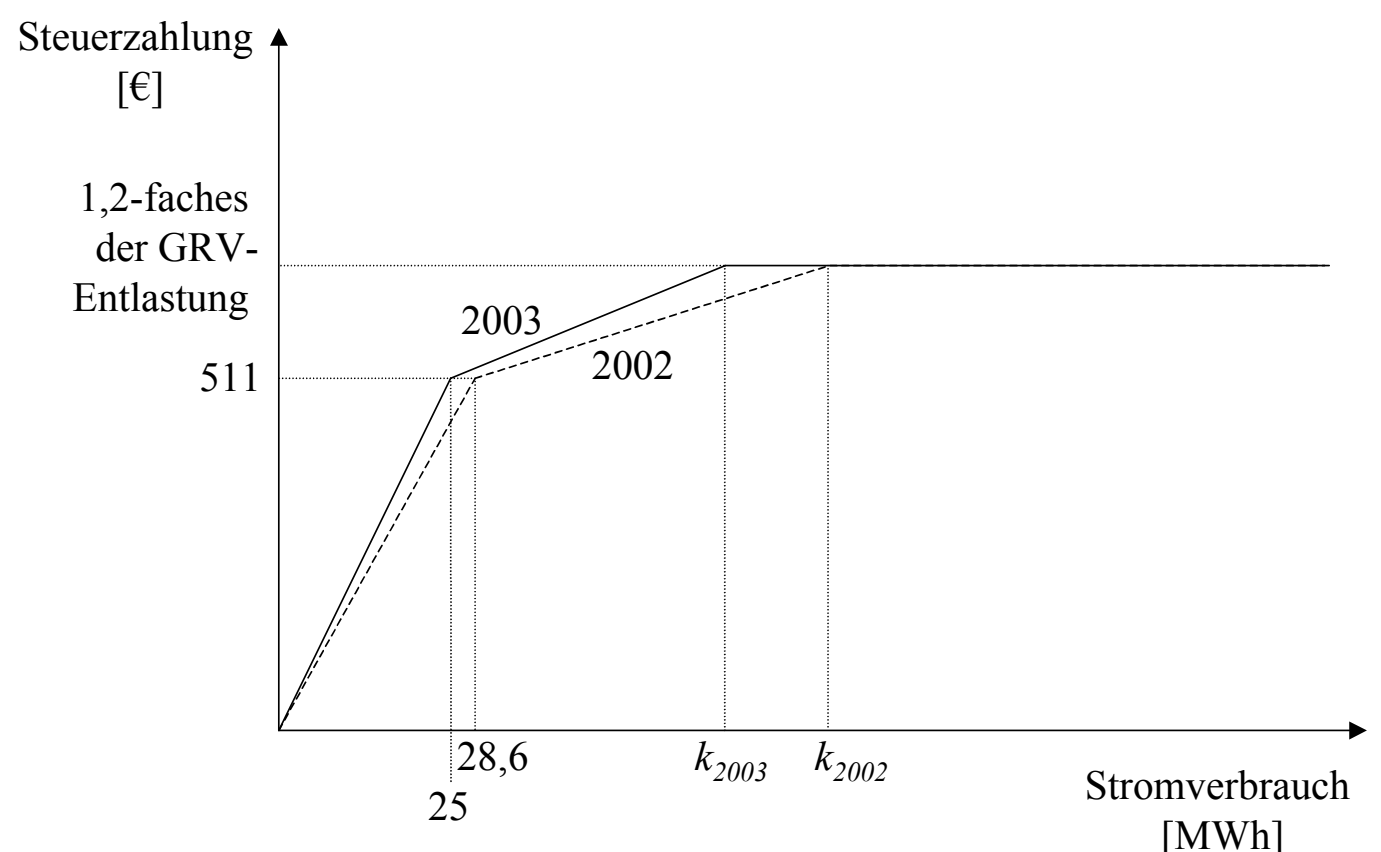

Abbildung 1: Der Tarif der Stromsteuer

Es wird davon ausgegangen, daß die Entlastung durch die Senkung der Rentenversicherungsbeiträge größer als $511 €$ ist. Bis zur Sockelmenge von 28,6 MWh steigt die Steuer entsprechend dem Normalsatz von 17,90 €/MWh steil an, danach nur noch mit 3,60 €/MWh. Bei der kritischen Verbrauchsmenge $k_{2002}$ erreicht die Steuer das 1,2-fache der Entlastung durch die Senkung der Rentenbeiträge, und bleibt von da an konstant. Der ökologische Anreiz ist deshalb auf die Unternehmen beschränkt, die weniger als diesen Schwellenwert verbrauchen, oder die ihren Energieverbrauch unter diesen Schwellenwert 
senken können. Durch die Erhöhung der Steuersätze verschärft jede weitere Stufe der ökologischen Steuerreform dieses Problem. Die durchgezogene Linie stellt die Steuerzahlung gemäß den für das Jahr 2003 vorgesehenen Sätzen von 20,50 €/MWh bzw. 4,10 €/MWh dar. Man erkennt, daß der dazugehörige kritische Wert $k_{2003}$ niedriger liegt. Das bedeutet, daß ein Unternehmen schon bei geringerem Stromverbrauch in den Genuß der Erstattung kommt als nach dem Tarif von 2002. Es wird im Jahre 2003 also mehr Unternehmen geben als 2002, für die der Anreiz zur Energieeinsparung wegfällt. Zwar wird für die verbleibenden, nicht energieintensiven Unternehmen dieser Anreiz durch den höheren Steuersatz verstärkt, aber es ist unklar, ob gesamtwirtschaftlich ein positiver Effekt verbleibt.

Bei näherer Betrachtung der Kompensationsregelung stößt man auf ein Detail, das zu unvermuteten Beschäftigungswirkungen führen kann. Es handelt sich dabei um die Vorschrift zur Berechnung der Entlastung, die ein Unternehmen durch die Senkung der Arbeitgeberanteile zur Rentenversicherung erfährt. Für Unternehmen, die nach 1998 gegründet wurden, ist diese Entlastung annähernd proportional zu den gezahlten Löhnen. Aufgrund der Kompensationsregelung entspricht die Belastung durch die Ökosteuern dem 1,2-fachen der so berechneten Entlastung. Wenn man die laufende Entlastung bei der Rentenversicherung saldiert, dann ergibt sich eine Nettobelastung aus ökologischen Steuern und Rentenversicherungsbeiträgen in Höhe des 0,2-fachen dieser Entlastung. Die Unternehmen können der Strom- und Mineralölsteuer deshalb ausweichen, indem sie die Lohnsumme senken, was jedoch einen Beschäftigungsabbau erfordert. Durch die Kompensationsregelung mutiert die Ökosteuer für neue Unternehmen also zu einer Beschäftigungssteuer, der man am besten durch Entlassungen begegnet. Die Ziele der ökologischen Steuerreform werden hier offenkundig auf den Kopf gestellt.

\section{Politökonomische Überlegungen}

Um die Diskrepanz zwischen theoretischem Ideal und praktischer Umsetzung der Ökologischen Steuerreform zu verstehen, muß man sich das Kalkül der politischen Entscheidungsträger vor Augen führen. Ende der 80er Jahre hatte das Thema Umweltpolitik einen relativen hohen Stellenwert in der öffentlichen Diskussion. Die von den Medien vermittelte Besorgnis der Bevölkerung über Umweltprobleme veranlaßte alle politischen Parteien dazu, der Umweltpolitik eine Priorität in ihren jeweiligen Wahlprogrammen 
einzuräumen. So rangierte beispielsweise das Thema Umweltschutz bis zur Rezession 1992/93 vor dem Problem der Arbeitslosigkeit (Politikbarometer 1989-1995). Nach dem Regierungswechsel 1998 kam es zum Einstieg in die Ökologische Steuerreform, einem Kernanliegen des grünen Koalitionspartners. Dabei wurde aber die konsequente Umsetzung der Ökologischen Steuerreform politökonomischen Überlegungen geopfert.

Es liegt in der Natur der Sache, daß einheitliche Umweltsteuern vor allem umweltintensive Sektoren belasten und dort zu spürbaren Gewinneinbußen und Beschäftigungsverlusten führen. In diesen Branchen würde der Verzicht auf Sonderregelungen die Steuermehrbelastung (Wünsche 1999, S. 201) und die Arbeitsplatzverluste (Böhringer und Rutherford 1997) vervielfachen. Von Ausnahmeregelungen profitieren diese Branchen deshalb in besonderem Maße, sodaß die potenziellen Erträge von Lobbying-Aktivitäten dort weit höher sind als in anderen Bereichen. Zudem sind die umwelt- bzw. energieintensiven Branchen in Deutschland als traditionelle Schlüsselindustrien gut organisiert und politisch einflußreich, was die Kosten von Lobbying senkt. Es ist deshalb nicht verwunderlich, daß gerade diese Branchen frühzeitig und mit Nachdruck für weitreichende Ausnahmeregelungen plädiert haben.

Die Politik hat sich diesen Begehrlichkeiten aus verschiedenen Gründen schnell gebeugt. Trotz hohem Umweltproblembewußtsein ist die Zahlungsbereitschaft für Umweltschutz in der Bevölkerung nur schwach ausgeprägt (ISSP 1993). Eine Ökologische Steuerreform findet deshalb nur dann eine breite Zustimmung, wenn sie praktisch nichts kostet. Als Reaktion hat die Politik die Aussicht auf eine zweite Dividende zunehmend betont und so die Bedeutung des Umweltziels relativiert. Allerdings scheint in der Bevölkerung die Skepsis bezüglich der Arbeitsmarkteffekte zu überwiegen. Dies ist nicht weiter verwunderlich. Augenfälligen Beschäftigungseinbrüchen in den energieintensiven Branchen stehen fragwürdige oder zumindest kaum wahrnehmbare Beschäftigungsgewinne in der übrigen Volkswirtschaft gegenüber. Damit ist das Beschäftigungsargument für den Politiker auch dann kaum nutzbar, wenn es auf gesamtwirtschaftlicher Ebene eine zweite Dividende geben sollte. Als Konsequenz wurde die Ökologische Steuerreform in Deutschland durch Ausnahmeregelungen verwässert, um spürbare Beschäftigungsverluste in energieintensiven Branchen zu vermeiden. Damit wurden zwar die politischen und ökonomischen Kosten der Ökologischen Steuerreform stark vermindert, gleichzeitig aber auch das Umweltziel praktisch aufgegeben. So sollte die Ökologische Steuerreform ursprünglich stark zur Verringerung des 
Treibhausgases $\mathrm{CO}_{2}$ beitragen. Jüngste Schätzungen der induzierten $\mathrm{CO}_{2}$-Minderung gehen aber nicht über 2-3 \% hinaus (Bach et al. 2001, S. 178).

Vor diesem Hintergrund stellt sich die Frage, warum die Ökologische Steuerreform in Deutschland bisher nicht offen aufgegeben wurde. Hier scheint die Koalitionsräson der rotgrünen Regierung ausschlaggebend zu sein. Für die Grünen gehört die Ökologische Steuerreform zum Glaubensbekenntnis. In Hinblick auf ihre Stammwählerschaft können sie sich ein Abrücken von der Ökologischen Steuerreform nicht leisten. Die SPD orientiert sich dagegen am Medianwähler, der eine Erhöhung von Umweltsteuern ablehnt, und zeigt deshalb wenig Interesse an einer konsequenten Umweltpolitik. Mit Rücksichtnahme auf den grünen Koalitionspartner kann sie zwar das Ökosteuer-Projekt nicht völlig abblasen, nimmt ihm aber mit weitreichenden Ausnahmeregelungen de facto die Wirkung.

Von der Idee einer Ökologischen Steuerreform ist nach ihrer Umsetzung in Deutschland nur noch ein ökologisch zahnloser Papiertiger geblieben. Dennoch erwartet der Bund für das Jahr 2003 ein zusätzliches Steueraufkommen in Höhe von 16,6 Mrd. € (Bach et al. 2001, S. 23). Davon entfallen 10,5 Mrd. $€$ auf die erhöhte Besteuerung von Kraftstoffen, sodaß die Ökologische Steuerreform im wesentlichen zu einer Benzinsteuer mutiert. Umgekehrt ist der Beitragssatz zur Rentenversicherung bisher nicht in dem Maße gesenkt worden wie angekündigt. Es drängt sich der Verdacht auf, daß neben Beschäftigungsaspekten der schiere Fiskalzweck die Ausgestaltung der Ökologischen Steuerreform dominiert. Dazu paßt auch, daß die Ökologische Steuerreform für energieintensive Unternehmen wie eine Pauschalsteuer wirkt. Dies ist umweltpolitisch verheerend, fiskalisch jedoch gerade erwünscht. In Anbetracht des Aufkommens verwundert es auch nicht, daß die Opposition für den Fall eines Wahlerfolgs zwar die im Jahre 2003 anstehende Stufe der Ökologischen Steuerreform wählerwirksam aussetzen möchte, aber die bisherigen Stufen kaum zurücknehmen wird.

\section{Schlußfolgerungen}

Die Ökologische Steuerreform wurde expressis verbis mit der Zielsetzung verabschiedet, einen ökologischen Umbau der Industriegesellschaft einzuleiten und den Arbeitsmarkt zu entlasten. Obwohl das Beschäftigungsziel in Zeiten hoher Arbeitslosigkeit Priorität genießt, ist seine Betonung im Zusammenhang mit der Ökologischen Steuerreform kontraproduktiv. 
Ökologisch sinnvolle Maßnahmen könnten gesellschaftspolitisch auf Ablehnung stoßen, weil die erhofften Arbeitsmarkteffekte ausbleiben. Da eine substantielle zweite Dividende unwahrscheinlich ist, kämen Umweltsteuern aus dieser Perspektive unter Rechtfertigungsdruck.

Gerade weil die Aussichten auf positive Beschäftigungswirkungen gering sind, ist das ökologische Ziel für die Begründung von Umweltsteuern ausschlaggebend. Umweltsteuern sollen die Umweltqualität erhöhen und müssen dementsprechend konzipiert werden. Eine einheitliche Besteuerung gewährleistet, daß alle Verursacher von Umweltverschmutzung mit den damit verbundenen Kosten konfrontiert werden. Dann wird Umweltschutz dort geleistet, wo er am wenigsten kostet. Aus theoretischer Sicht können Abweichungen von dieser einfachen Regel vorteilhaft sein, wenn globale Umweltexternalitäten im nationalen Alleingang gemindert werden sollen oder wenn Verzerrungen durch andere Steuern bestehen. Für die Praxis sind die aus der Theorie ableitbaren Empfehlungen zur Steuerdifferenzierung jedoch kaum relevant.

Die gegenwärtige Ausgestaltung der Ökosteuer weicht vom Prinzip der einheitlichen Besteuerung erheblich ab. Über weitreichende Ausnahmen wird die ökologische Lenkungswirkung stark abgeschwächt. Umweltschutz wird damit teurer, als er aus gesamtwirtschaftlicher Sicht sein müßte.

Die Verwässerung der Ökologischen Steuerreform ist politisch auf die geringe Zahlungsbereitschaft der Wähler für Umweltschutz und die starke sektorale Konzentration von Anpassungskosten einheitlicher Umweltsteuern zurückzuführen. Ausnahmeregelungen für energieintensive Branchen haben die politischen und ökonomischen Kosten der Ökologischen Steuerreform verringert. Damit wurde aber auch das Umweltziel faktisch aufgegeben.

Welche Perspektiven hat die Ökologische Steuerreform? Klar ist, daß eine konsequent verfolgte Ökologische Steuerreform zu Anpassungsproblemen für umweltintensive Branchen führen wird. Dieser Strukturwandel ist zur Verminderung von Umweltbelastungen erforderlich und muß bei gesellschaftspolitischem Konsens über die $\mathrm{zu}$ erreichenden Umweltqualitätsziele auch hingenommen werden. Es scheint aber so, daß der Wähler nicht bereit ist, die Kosten eines weitreichenden Umweltschutzes zu tragen. Dies muß bei der 
gesellschaftspolitischen Entscheidung über das Ausmaß von Umweltschutz berücksichtigt werden. Eine seriöse Politik steht vor zwei Alternativen. Entweder sie folgt der Mehrheit der Wähler, die eine konsequente Umweltpolitik ablehnt, und gibt die Ökologische Steuerreform offen auf. Oder sie setzt die Ökologische Steuerreform ohne Ausnahmen um und wirbt dafür um politische Mehrheiten.

\section{Literaturverzeichnis}

Bach, S., C. Bork, M. Kohlhaas, C. Lutz, B. Meyer, B. Praetorius und H. Welsch (2001), Die Ökologische Steuerreform in Deutschland: Eine modellgestützte Analyse ihrer Wirkungen auf Wirtschaft und Umwelt, Physica-Verlag, Heidelberg.

Barrett, S. (1994), Self-Enforcing Environmental Agreements, Oxford Economic Papers 46, S. $804-878$.

Böhringer, C. (2002), Environmental Tax Differentiation between Industries and Households - Implications for Efficiency and Employment, ZEW discussion paper 02-08, Mannheim.

Böhringer, C. und T.F. Rutherford (1997), Carbon Taxes with Exemptions in an Open Economy - A General Equilibrium Analysis of the German Tax Initiative, Journal of Environmental Economics and Management 32, 189-203.

Bolton, G.E. und A. Ockenfels (2000), ERC : A Theory of Equity, Reciprocity, and Competition, The American Economic Review 90, S. 166-193.

Goulder, L. H. (1995), Environmental Taxation and the Double Dividend: A Readers' Guide. International Tax and Public Finance 2, 157-183.

Hoel, M (1996), Should a Carbon Tax be Differentiated across Sectors?, Journal of Public Economics 59, S. 17-32. 
IPCC (2001), Climate Change 2001: Mitigation, Contribution of Working Group III to the Third Assessment Report of the Intergovernmental Panel on Climate Change, Cambridge University Press, Cambridge.

ISSP (1993), Environment I, Codebook ZA Study 2450, Zentralarchiv für Empirische Sozialforschung, Köln.

Lange, A. und C. Vogt (2002), Cooperation in International Environmental Negotiations due to a Preference for Equity, erscheint in: Journal of Public Economics.

Oliveira-Martins, J., H.M. Burniaux und J.P. Martin (1992), Trade and the Effectiveness of Unilateral $\mathrm{CO}_{2}$ Abatement Policies: Evidence from GREEN, OECD Economic Studies 19, Paris.

Politikbarometer (1989-1995), Zentralarchiv für Empirische Sozialforschung, Köln, ZA-Nr. 1779, 1920, 2102, 2275, 2378, 2546, 2765.

Wissenschaftlicher Beirat beim Bundesministerium der Finanzen (1997), Umweltsteuern aus finanzwissenschaftlicher Sicht, Schriftenreihe des Bundesministeriums der Finanzen 63, Stollfuss, Bonn.

Wünsche, A. (1999), Umweltabgaben und Unternehmensbesteuerung: Eine nationale und internationale Analyse der Belastungs- und Entscheidungswirkungen, ZEWWirtschaftsanalysen Band 38, NOMOS, Baden-Baden.

Gesetz zum Einstieg in die ökologische Steuerreform, Bundesgesetzblatt I, S. 378, 1999.

Gesetz zur Fortführung der ökologischen Steuerreform, Bundesgesetzblatt I, S. 2432, 1999. 